\title{
Study of a Car Body Tilting System Using a Variable Link Mechanism: Fundamental Characteristics of Pendulum Motion and Strategy for Perfect Tilting*
}

\author{
Hidehisa YOSHIDA $^{* *}$ and Masao NAGAI ${ }^{* * *}$ \\ **National Defense Academy of Japan, \\ Hashirimizu 1-10-20, Yokosuka-shi, Kanagawa-Pref, 239-8686, Japan \\ E-mail: yoshida@nda.ac.jp \\ ***Tokyo University of Agriculture \& Technology, \\ Naka-cho 2-24-16, Koganei-shi, Tokyo, 184-8588, Japan
}

\begin{abstract}
This paper analyzes the fundamental dynamic characteristics of a tilting railway vehicle using a variable link mechanism for compensating both the lateral acceleration experienced by passengers and the wheel load imbalance between the inner and outer rails. The geometric relations between the center of rotation, the center of gravity, and the positions of all four links of the tilting system are analyzed. Then, equations of the pendulum motions of the railway vehicle body with a four-link mechanism are derived. A theoretically discussion is given on the geometrical shapes employed in the link mechanism that can simultaneously provide zero lateral acceleration and zero wheel load fluctuation. Then, the perfect tilting condition, which is the control target of the feedforward tilting control, is derived from the linear equation of tilting motion.
\end{abstract}

Key words: Railway, Dynamics of Machinery, Motion Control, Tilting Control, Link Mechanism, Vehicle Dynamics

\section{Introduction}

When a railway vehicle runs on a curved section of track, the cant alone is unable to compensate for the over-centrifugal acceleration generated by excessive speed, and the riding comfort of passengers readily deteriorates. In this case, it is necessary for the effect of over-centrifugal acceleration that affects the car body to be reduced by controlling the tilt.

In many tilt-control systems, the tilt angle is controlled by altering the center of gravity of the car body. However, the center of gravity moves to the outside rail during turning, and thus the creation of an imbalance between the wheel loads of the right- and left-hand sides, which support the car body, is unavoidable.

A car body tilt-control system with a link mechanism was used in the present study to investigate the possibility of compensating both over centrifugal acceleration and wheel load imbalance; the optimal link shape was determined to this end.

Since the tilting motion of linked car bodies includes a strong nonlinear component, conventional analysis of the tilting mechanism relies on the use of computer-aided software engineering (multi-body analysis) that is based on numerical calculations, rather than a fully analytical method. However, this type of numerical analysis is not suitable for theoretical

Received 31 Oct., 2006 (No. T-06-0176) Japanese Original : Trans. Jpn. Soc. Mech. Eng., Vol. 72, No. 721, C (2006), pp.2748-2755 (Received 18 Jan., 2006) [DOI: 10.1299/jsdd.1.200] 
tilting control.

The present study introduces a theoretical analysis formulation of the car body tilting system having a four-link mechanism, derived from experimental results. Next, the results of a scaled-model experiment are compared with the analytical results of the theoretical model by using an approximation technique, and the validity of the theoretical analytical model is evaluated by analyzing the natural frequency of free vibration.

Finally, a new tilting system that compensates both the lateral acceleration that passengers experience and the wheel load imbalance between the inner and outer rails is proposed.

\section{Model of Link Mechanism}

\subsection{Assumptions of the model}

Figure 1 shows the cross-sectional model of the titling railway vehicle, which consists of the target body, a swing bolster, a truck-wheelset, and the rail system. In order to clarify the basic movement of the vehicle, the spring elements (such as air springs) are not taken into consideration. Rather, the relative movement between the body- and swing bolster is neglected, and it is assumed that the body and swing bolster move in unison. Hereafter in this report, the body and swing bolster are collectively referred to as "the body".

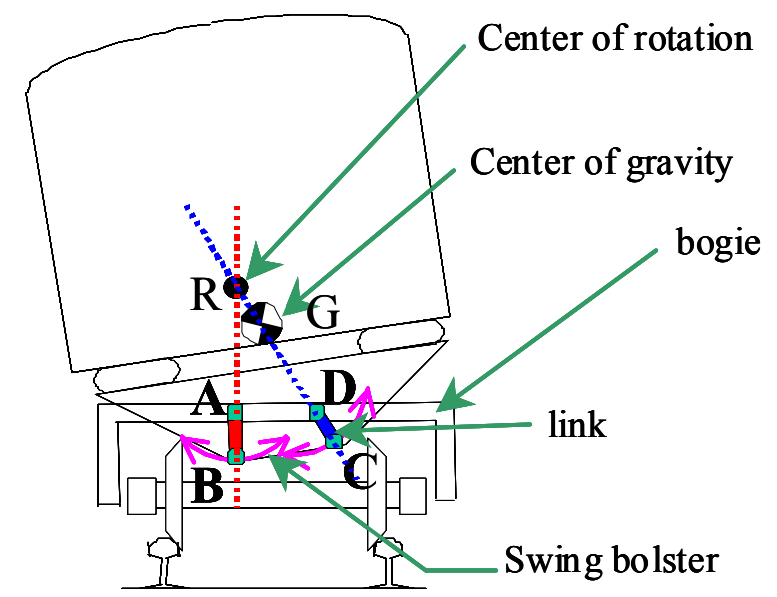

Fig. 1 Cross-section model of tilting vehicle using link mechanism

Moreover, if it is assumed that the truck, and the wheelset, and the rail system are connected and under go the same movement, it will be possible to deal with only the tilting characteristics of the pure link mechanism, which supports the body by a truck.

The body $\mathbf{B C}$ and the truck $\mathbf{A D}$ are connected by two links, and these links constitute a four-link system with pendulum motion for the tilting of the vehicle. Although $\mathbf{B}[\mathbf{C}]$ rotates around a center $\mathbf{A}[\mathbf{D}]$, since the length of the body $\mathbf{B C}$ is fixed, tilting of the body occurs. The crossing point on the extended lines from links $\mathbf{A B}$ and $\mathbf{C D}$ is defined as the rotational center $\mathbf{R}$.

Figure 2 shows the geometric characteristics of the link mechanism. Since it becomes parallel [ $\mathbf{B C}$ and $\mathbf{A D}$ ] at when $\boldsymbol{r}_{\mathrm{b}}=\boldsymbol{r}_{\mathrm{d}}$, the rotation center $\mathbf{R}$ does not exist. Therefore, the condition $\boldsymbol{r}_{\mathbf{b}}=\boldsymbol{r}_{\mathrm{d}}$ does not influence the model. Since the rotation center $\mathrm{R}$ is an intersection on the extended line of link $\mathbf{A B}$ and link $\mathbf{C D}$, the coordinates_of point $\mathbf{R}$ are reached by the formulations of link $\mathbf{A B}$ and $\mathbf{C D}$.

Assuming that truck $\mathbf{A D}$ is parallel to the Y-axis, the coordinates of point $\mathbf{A}$ are $\left(y_{\mathrm{a}}, z_{\mathrm{a}}\right)$. In addition, the external angle between link $\mathbf{A B}$ and truck $\mathbf{A D}$ is represented by $\theta_{\mathrm{a}}$, and the tilt angle of the car body for the truck is represented by $\theta_{b}$. In order to simplify the model, the link lengths of the right- and left-hand sides are assumed to be identical $\left(\boldsymbol{r}_{\mathbf{a}}=\boldsymbol{r}_{\mathrm{c}}\right)$. Hence, 
using the coordinates of the center of rotation $\mathbf{R}(y, z)$, the following equations are derived

$$
\begin{aligned}
& y=y_{a}+r_{d} \cos \theta_{a} \frac{r_{a} \sin \theta_{a}-r_{b} \sin \theta_{b}}{r_{b} \sin \left(\theta_{a}-\theta_{b}\right)-r_{d} \sin \theta_{a}} \\
& z=z_{a}+r_{d} \sin \theta_{a} \frac{r_{a} \sin \theta_{a}-r_{b} \sin \theta_{b}}{r_{b} \sin \left(\theta_{a}-\theta_{b}\right)-r_{d} \sin \theta_{a}}
\end{aligned}
$$

The center of gravity of the car body $\mathbf{G}$ is assumed to deviate from the car body $\mathbf{B C}$ as the value of angle $\alpha$. In addition, the center of gravity of the car body $\mathbf{G}$ is perpendicular to the car body BC. The following equations are then derived using the coordinates of the center of gravity of the car body $\mathbf{G}\left(y_{\mathrm{g}}, z_{\mathrm{g}}\right)$.

$$
\begin{aligned}
& y_{g}=y_{a}-r_{a} \cos \theta_{a}+r_{e} \cos \left(\theta_{b}+\alpha\right) \\
& z_{g}=z_{g}-r_{a} \sin \theta_{a}+r_{e} \sin \left(\theta_{b}+\alpha\right)
\end{aligned}
$$

These coordinates are expressed as a function of the car body tilt angle $\theta_{b}$.

$$
\theta_{a}=\tan ^{-1}\left(\frac{r_{b} \sin \theta_{b}}{r_{b} \cos \theta_{b}-r_{d}}\right)+\cos ^{-1}\left(\frac{\sqrt{r_{b}^{2}+r_{d}^{2}-2 r_{b} r_{d} \cos \theta_{b}}}{2 r_{a}}\right)
$$

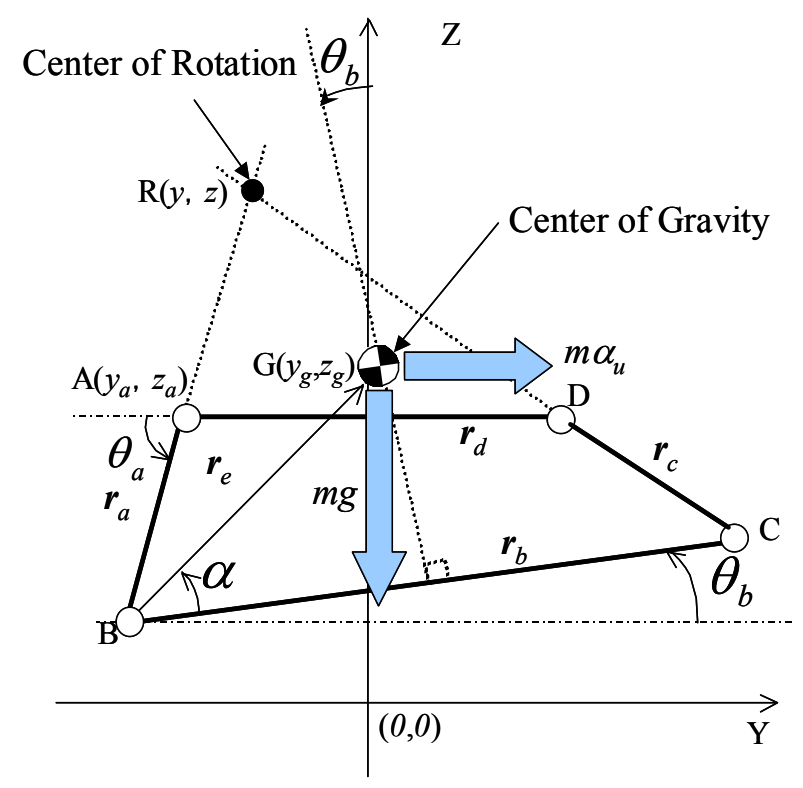

Fig. 2 Geometrical model of the four link mechanism

\subsection{Tilting model}

This section considers the case when an external force acts on the center of gravity $\mathbf{G}$ supported by the link, and the center of rotation $\mathbf{R}$ is also variable so that the link motion can be approximated as pendulum motion. Furthermore, in the present study it is assumed that the rotational motion of this system behaves as a simple pendulum, as shown in Fig. 3.

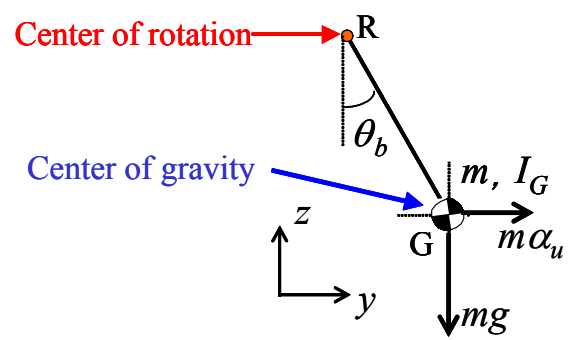

Fig. 3 Link mechanism and a simple pendulum 
Defining gravity $\mathbf{g}$ over the centrifugal acceleration $\alpha_{u}$, which affects the center of gravity of the car body, the car body mass $\boldsymbol{m}$, and the moment of inertia $\boldsymbol{I}_{\mathrm{G}}$ around the center of gravity of the car body, based on the balancing moment using the principle of the virtual work, the equation of motion for the car body tilt angle $\theta_{b}$ can be expressed as follows:

$$
\begin{aligned}
\left\lfloor I_{G}+m\left\{\left(y_{g}-y\right)^{2}\right.\right. & \left.\left.+\left(z_{g}-z\right)^{2}\right\}\right] \ddot{\theta}_{b}= \\
& -m g\left(y_{g}-y\right)-m \alpha_{u}\left(z_{g}-z\right)
\end{aligned}
$$

This equation is that for a simple pendulum in which the moment of inertia, which corresponds to the arm length of the simple pendulum, varies with $\theta_{b}$. Using Eqs. (1) to (6), it is possible to solve the motion of $\theta_{b}$.

\subsection{Linear tilting model}

This equation for the tilting motion is not appropriate for analysis because it is nonlinear. When the controller is designed, the equation of a linear system with the good prospect is desirable. So we use a linear approximation of the equation for tilting motion. Since it is assumed that $\theta_{\mathrm{b}}$ oscillates around the zero-center in the car body tilting model, the following three items were linearized to the first approximation by using Maclaurin's theorem.

(1) $\theta_{a}$ in Eq. (5):

$$
\begin{aligned}
& \theta_{a}=\theta_{a}\left(\theta_{b}\right)=\theta_{a}(0)+\left.\frac{d \theta_{a}}{d \theta_{b}}\right|_{\theta_{b}=0} \theta_{b} \\
& \theta_{a}=\left\{\frac{\pi}{2}-\sin ^{-1}\left(\frac{r_{b}-r_{d}}{2 r_{a}}\right)\right\}+\frac{r_{b}}{r_{b}-r_{d}} \theta_{b}
\end{aligned}
$$

(2) Distance between the center of rotation $\mathrm{R}$ and the center of gravity $\mathrm{G}$ :

$$
\begin{aligned}
& y_{g}-y=\bar{A}_{0}+\left(\bar{B}_{0}-\bar{C}_{0}\right) \theta_{b} \\
& z_{g}-z=\bar{C}_{0}+\bar{A}_{0} \theta_{b} \\
& \bar{A}_{0}=-\frac{r_{b}}{2}+r_{d} \cos \alpha \\
& \bar{B}_{0}=\frac{2 r_{a}^{2} r_{b} r_{d}}{\left(r_{b}-r_{d}\right)^{2} \sqrt{4 r_{a}^{2}-\left(r_{b}-r_{d}\right)^{2}}} \\
& \bar{C}_{0}=r_{e} \sin \alpha-\frac{r_{b} \sqrt{4 r_{a}^{2}-\left(r_{b}-r_{d}\right)^{2}}}{2\left(r_{b}-r_{d}\right)}
\end{aligned}
$$

(3) Moment of inertia $I_{\mathrm{G}}$

The car body tilt angle $\theta_{b}$ is assumed to be zero.

$$
I_{G}+m\left\{\left(y_{g}-y\right)^{2}+\left(z_{g}-z\right)^{2}\right\}=I_{G}+m\left(\bar{A}_{0}^{2}+\bar{C}_{0}^{2}\right)
$$

These equations indicate that it is possible that the equation of car body tilting motion by the link mechanism is similar to the equation of motion of a linear rotating system.

$$
\begin{aligned}
& {\left[I_{G}+m\left(\bar{A}_{0}^{2}+\bar{C}_{0}^{2}\right)\right] \ddot{\theta}_{b}=} \\
& \quad-m\left(g \bar{A}_{0}+\alpha_{u} \bar{C}_{0}\right)-m\left(\alpha_{u} \bar{A}_{0}+\bar{B}_{0}-g \bar{C}_{0}\right) \theta_{b}
\end{aligned}
$$

\subsection{Linear and nonlinear equation of tilting motion}

The nonlinear equation of tilting motion $E q$. (6) is compared with the linear equation of tilting motion Eq. (14). Figure 4(a) shows the relationship between the nonlinear and linear moments of inertia. Each equation is transformed, and the relationship between the angle 
and the angular acceleration is analyzed and shown in Fig. 4(b). In addition, using the parameter values $\boldsymbol{r}_{\mathrm{d}}=0.250 \mathrm{~m}, \boldsymbol{r}_{\mathrm{b}}=0.350 \mathrm{~m}$, and $\boldsymbol{r}_{\mathrm{a}}=\boldsymbol{r}_{\mathrm{c}}=0.250 \mathrm{~m}$, which are described later for the experimental device.

$$
\begin{aligned}
& \ddot{\theta}_{b}=\frac{-m g\left(y_{g}-y\right)-m \alpha_{u}\left(z_{0}-z\right)}{I_{G}+m\left\{\left(y_{g}-y\right)^{2}+\left(z_{0}-z\right)^{2}\right\}} \\
& \ddot{\theta}_{b}=\frac{-m\left(g \bar{A}_{0}+\alpha_{u} \bar{C}_{0}\right)}{I_{G}+m\left(\bar{A}_{0}{ }^{2}+\bar{C}_{0}{ }^{2}\right)}-\frac{m\left(\alpha_{u} \bar{A}_{0}+\bar{B}_{0}-g \bar{C}_{0}\right) \theta_{b}}{I_{G}+m\left(\bar{A}_{0}{ }^{2}+\bar{C}_{0}{ }^{2}\right)}
\end{aligned}
$$

Based on these results, the approximation is accurate for a car body tilt angle $\theta_{\mathrm{b}}$ of up to 10 degrees. Since the present study focuses on the control for the case when the tilt angle does not exceed 10 degrees, the theoretical analysis in the next chapter is performed using this approximate equation of motion.

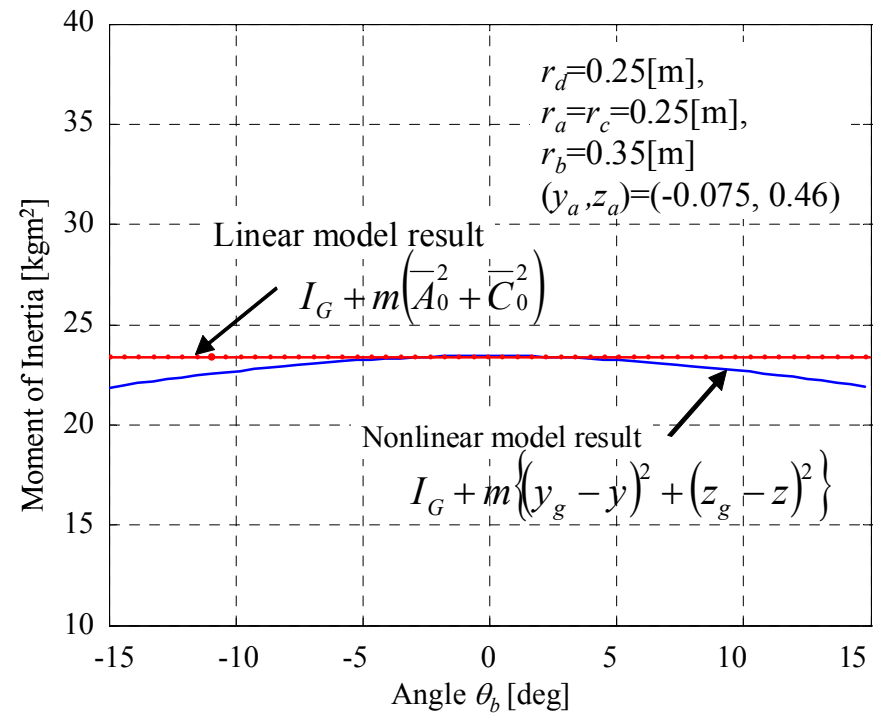

(a) Relationship between moment of inertia and angle

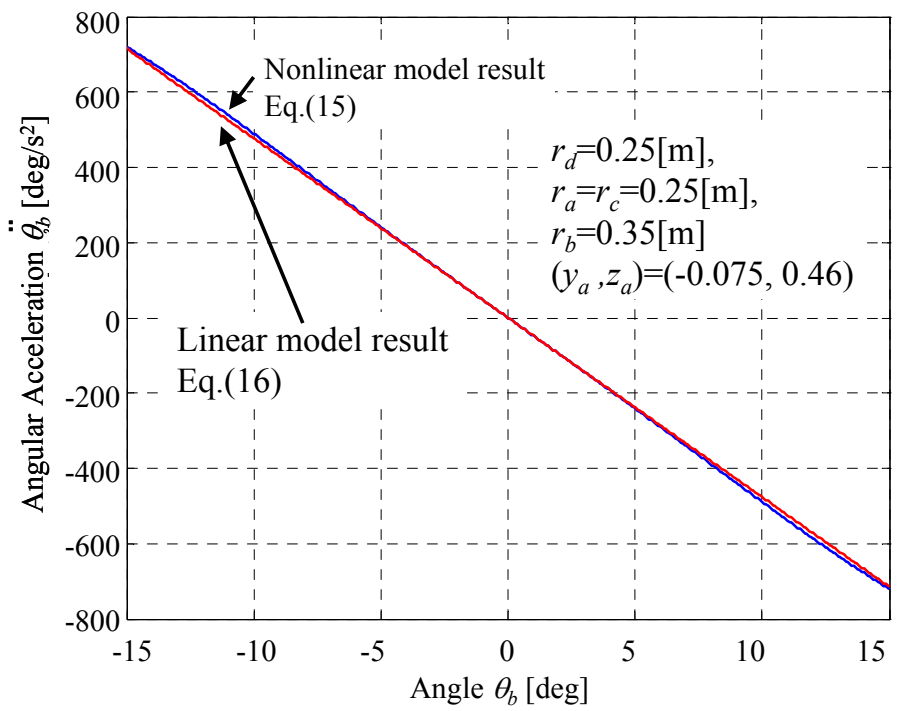

(b) Relationship between angular acceleration and angle

Fig.4 Results of linear model and nonlinear model 


\section{Natural Pendulum Characteristics}

\subsection{Experimental setup}

In the present study, an experiment was performed in order to examine the modeling of the movement of a car body with link behavior when moving along a curved track. An overview of the tilting experiment setup is shown in Fig. 5.

The over-centrifugal acceleration is simulated by tilting a table placed under the experimental apparatus. Lengths $\boldsymbol{r}_{\mathrm{d}}$ is adjustable, while length $\boldsymbol{r}_{\mathrm{b}}$ is continuously adjustable.

The free vibration is generated by centrifugal force and gravity, when the tilting car travels along the curved section. In addition, the tilt angle is balanced in the static condition by the centrifugal force and gravity on the curve. As a characteristic under these natural pendulum conditions, the three items described below are examined.

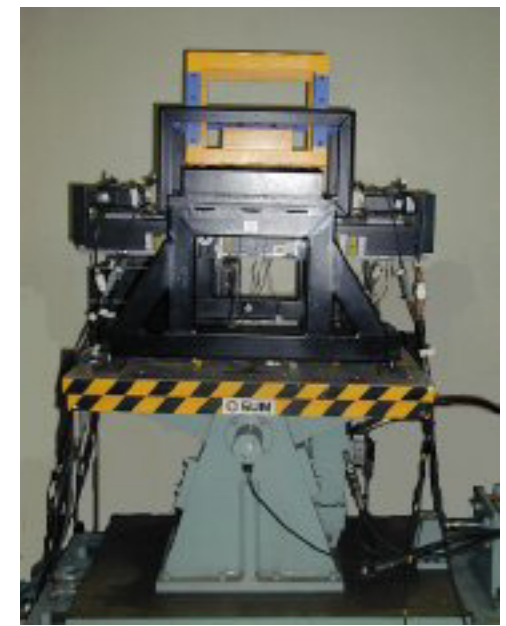

(a) Outside view (Photograph)

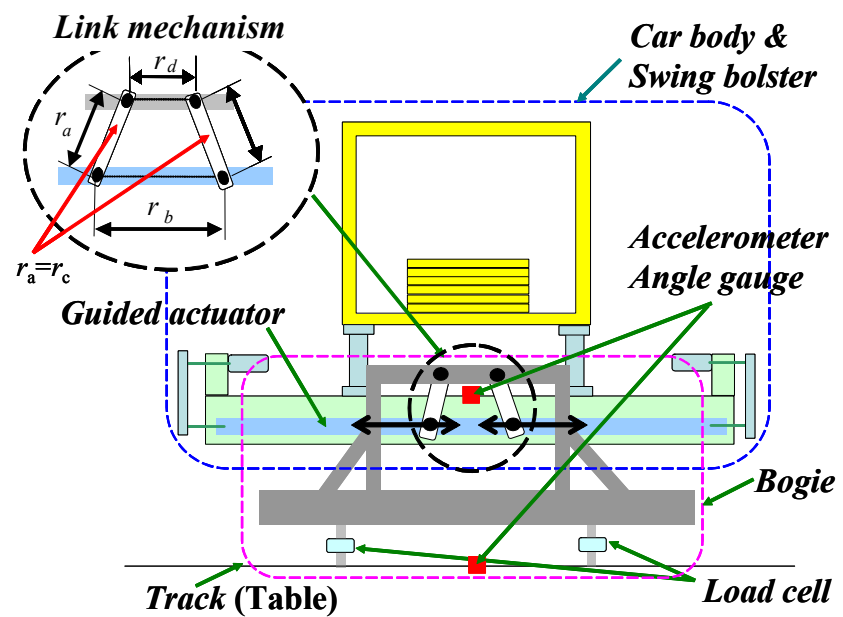

(b) Outline of tilting experimental model with sensors
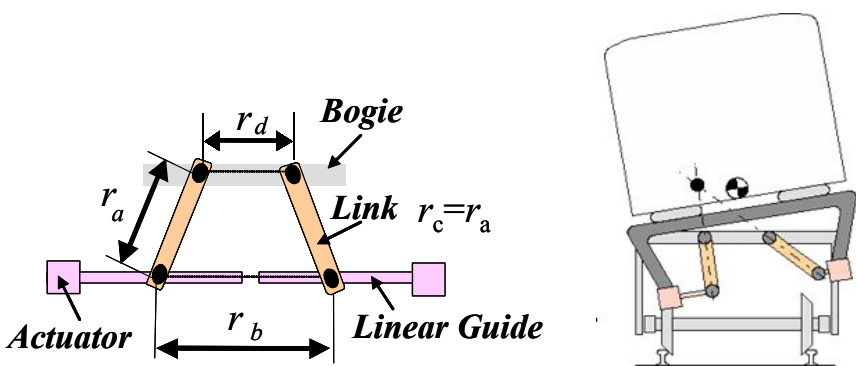

(c) Outline of tilting model with link

Fig. 5 Experimental setup 


\subsection{Natural frequency}

From $E q$. (14), the natural frequency is given as follows:

$$
f_{n}=\frac{1}{2 \pi} \sqrt{\frac{m\left(\alpha_{u} \bar{A}_{0}+\bar{B}_{0}-g \bar{C}_{0}\right)}{I_{G}+m\left(\bar{A}_{0}^{2}+\bar{C}_{0}^{2}\right)}}
$$

The theoretical result (Eq. (17)) and the experimental result of natural frequency $f_{\mathrm{n}}$ are shown in Fig. 6. When $\boldsymbol{r}_{\mathrm{b}}$ is between $0.250 \mathrm{~m}$ and $0.100 \mathrm{~m}$, both the theoretical and experimental results are similar. This thus confirms that the theoretical equation of the tilting motion is valid.

When the length $\boldsymbol{r}_{\mathrm{d}}$ is $0.100 \mathrm{~m}$, the tendency for the natural frequency to decrease as $\boldsymbol{r}_{\mathrm{b}}$ increases can be seen from the theoretical results. When the length $\boldsymbol{r}_{\mathrm{d}}$ is $0.250 \mathrm{~m}$, the natural frequency increases as $\boldsymbol{r}_{\mathrm{b}}$ increases. In particular, the natural frequency in the case of an inverse-trapezoid shape as $\boldsymbol{r}_{\mathrm{b}}<\boldsymbol{r}_{\mathrm{d}}$, is small, and in the positive trapezoid state as $\boldsymbol{r}_{\mathrm{b}}>\boldsymbol{r}_{\mathrm{d}}$, the natural frequency becomes larger, until it saturates at a certain value of $\boldsymbol{r}_{\mathrm{b}}$.

\subsection{Car body lateral acceleration in the steady state curve}

When the over-centrifugal acceleration $\alpha_{u}$ affects the car body, it is possible to compensate the over-centrifugal acceleration $\alpha_{u}$ by tilting the car body. The lateral acceleration $\alpha_{d}$ that the passenger experiences in the car, is expressed by the following equation, under the assumption that the tilt angle is small:

$$
\begin{aligned}
\alpha_{d} & =\rho_{0} V^{2} \cos \left(\theta_{b}+\theta_{0}\right)-g \sin \left(\theta_{b}+\theta_{0}\right) \\
& \approx \alpha_{u}-g \theta_{b}
\end{aligned}
$$

where $V$ is the curve passage speed, $\rho$ is the curvature, and $\theta_{0}$ is the cant angle.

The tilting angle is balanced in the static condition by centrifugal force and gravity on the curve. It is possible to calculate the car body tilt angle $\theta_{00}$ using $E q$. (14).

$$
\theta_{b 0}=-\frac{g \bar{A}_{0}+\alpha_{u} \bar{C}_{0}}{\alpha_{u} \bar{A}_{0}+g \bar{B}_{0}-g \bar{C}_{0}}
$$

Therefore, the lateral acceleration $\alpha_{\mathrm{d} 0}$ that the passenger experiences in the car, for a steady state curve is given by the following equation:

$$
\alpha_{d 0}=\alpha_{u}+\frac{g \bar{A}_{0}+\alpha_{u} \bar{C}_{0}}{\alpha_{u} \bar{A}_{0}+g \bar{B}_{0}-g \bar{C}_{0}} g
$$

Figure 7 shows the lateral acceleration $\alpha_{d}$ on a steady state curve as a function of $\boldsymbol{r}_{\mathrm{b}}$. In theory and the experimental, the same minimum tendency is observed for $\boldsymbol{r}_{\mathrm{b}}$, and the values are also approximately equal. The relational expression in the steady state is thus valid.

\subsection{Wheel load ratio in the steady state curve}

When over-centrifugal acceleration $\alpha_{u}$ acts on the car body, the wheel loads of the rightand left-hand sides, which the rail receives from the wheel, fluctuates. It is possible to calculate this wheel load fluctuation by balancing the moment. The wheel load fluctuation is shown by the wheel load ratio $\Delta \boldsymbol{P} / \boldsymbol{P}_{0}$ that the fluctuated value $\Delta \boldsymbol{P}$, which is charged on the wheel, divided by the static wheel load $\boldsymbol{P}_{0}$. Here $m_{\mathrm{t}}$ is the car truck mass, $\left(y_{\mathrm{t}}, z_{\mathrm{t}}\right)$ are the coordinates of the center of gravity of the truck, $h_{\mathrm{t}}$ is the height from the track to the center of gravity of the truck, $r_{\mathrm{e}}$ is the length $\mathbf{B G}$, and $G$ is the track gage. The wheel load ratio under the steady state curve is shown as the following Maclaurin theorem of first approximation. 


$$
\begin{gathered}
\Delta P / P_{0}=\frac{2\left(m y_{g} g+m z_{g} \alpha_{u}+2 m_{t} y_{t} g+2 m_{t} z_{t} \alpha_{u}\right)}{G g\left(m+2 m_{t}\right)} \\
=\frac{2 \bar{D}_{0}}{G g\left(m+2 m_{t}\right)}+\frac{2 m\left(\alpha_{u} \bar{A}_{0}-g \bar{C}_{0}\right)}{G g\left(m+2 m_{t}\right)} \theta_{b} \\
\bar{D}_{0}=m\left\{\bar{A}_{0} g+\left(z_{a}-\frac{\sqrt{4 r_{a}^{2}-\left(r_{b}-r_{d}\right)^{2}}}{2}+r_{e} \sin \alpha\right) \alpha_{u}\right\} \\
+2 m_{t} h_{t} \alpha_{u}\left(1+\sqrt{a+\left(\frac{\alpha_{u}}{g}\right)^{2}}\right)
\end{gathered}
$$
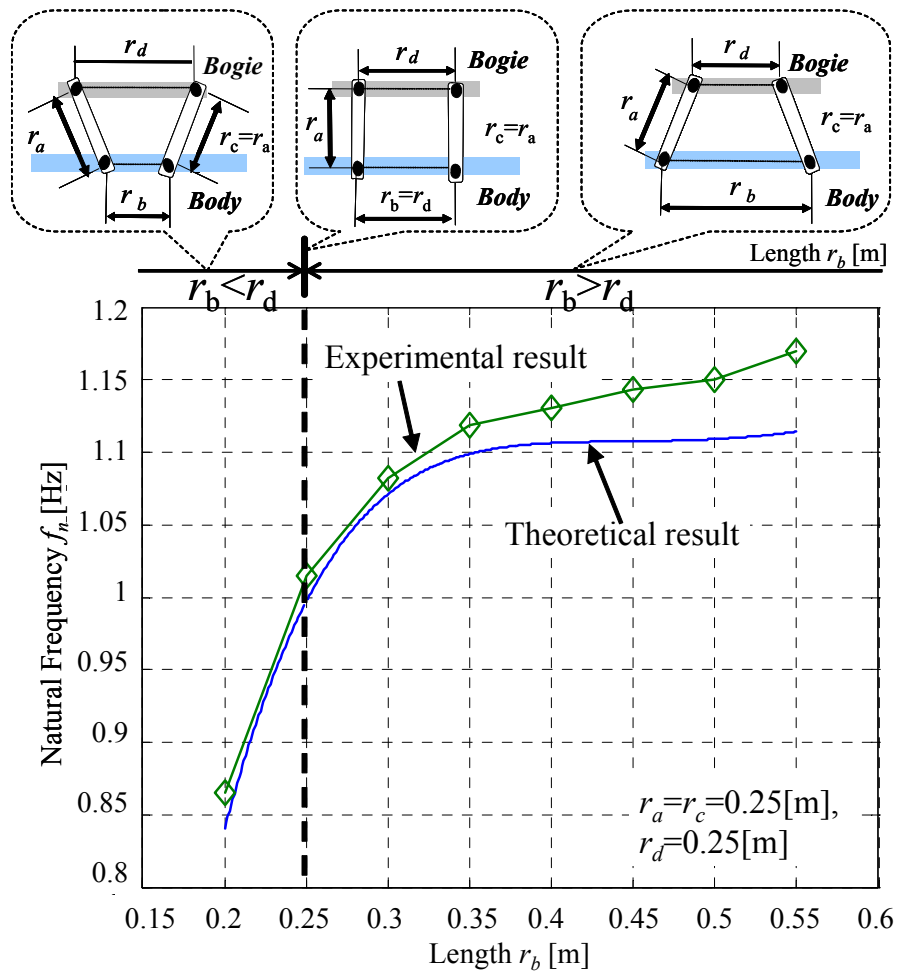

Fig. 6 Natural frequency characteristics

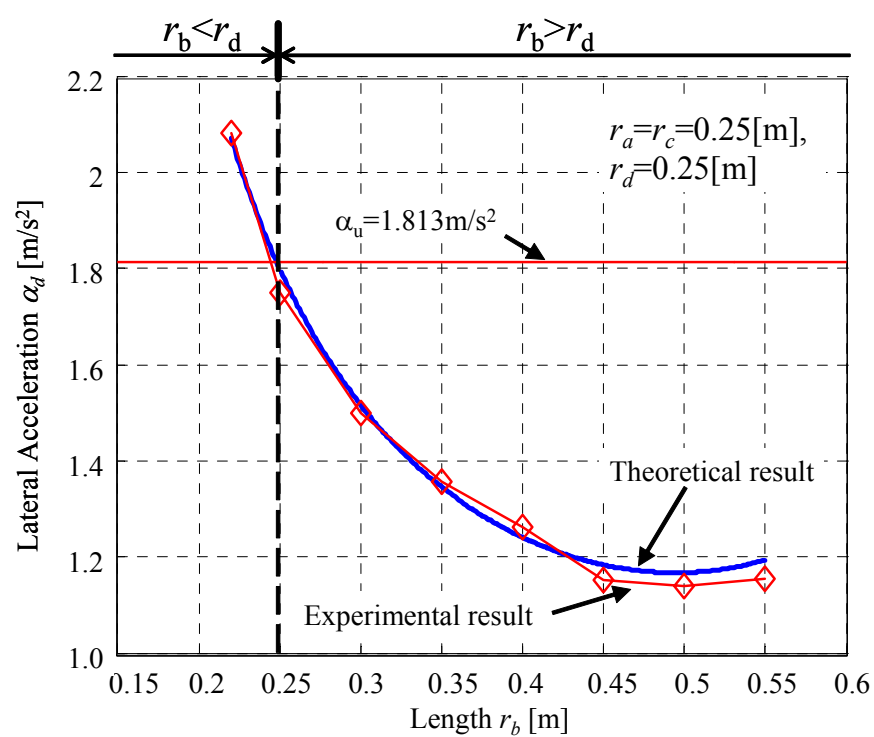

Fig. 7 Steady-state lateral acceleration for passengers on curve 
Figure 8 shows the results of the wheel load ratio $\Delta \boldsymbol{P} / \boldsymbol{P}_{0}$ on the steady state curve. The tendency and value of both the theoretical and experimental results are in reasonable agreement, confirming the validity of the analytical expression of the wheel load ratio. The effect of wheel load ratio, compared with natural frequency and over-centrifugal acceleration, by changing of the link shape is little, and it is shown that, under this condition, it is difficult to reduce the wheel load ratio to approximately zero.

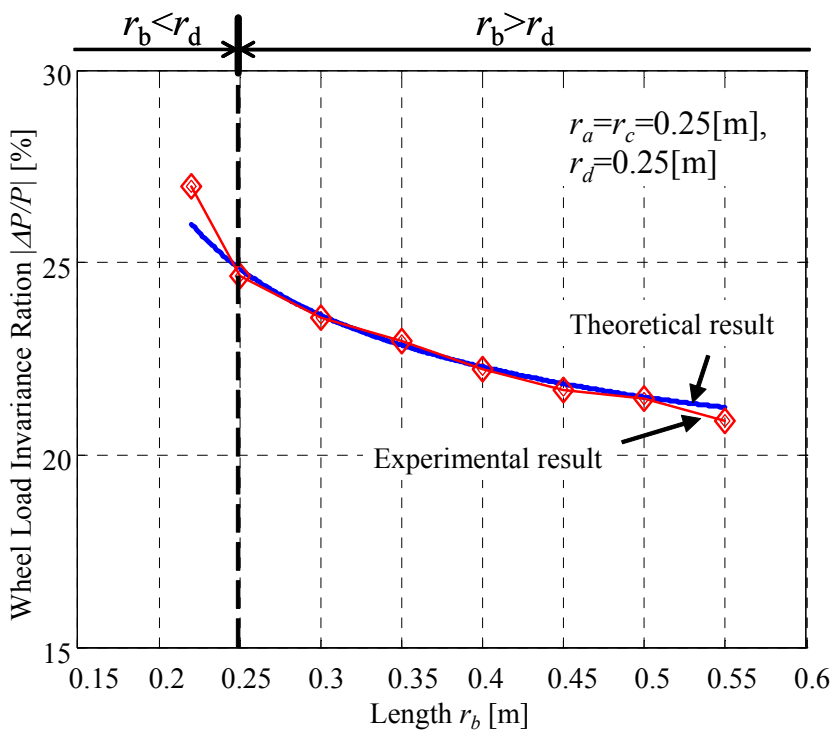

Fig. 8 Steady-state wheel load ratio on the steady state curve

\section{New Strategy of Tilting Control}

This section discusses the desired titling angle, at which both lateral acceleration and wheel load ratio are compensated to the extent possible, when an over-centrifugal lateral acceleration acts on the car body.

\subsection{Proposal of new tilting control input}

It is not possible to obtain sufficient tilting performance, although the swing of the natural pendulum using the usual link system, as shown by the theoretical analysis model in Section 3. Therefore, in the conventional tilting system using the link mechanism, the outside actuator is operated in order to maintain the performance of the required tilt angle. On the other hand, the present study proposes a new tilting control strategy using the system, the length of each link of which is completely changeable.

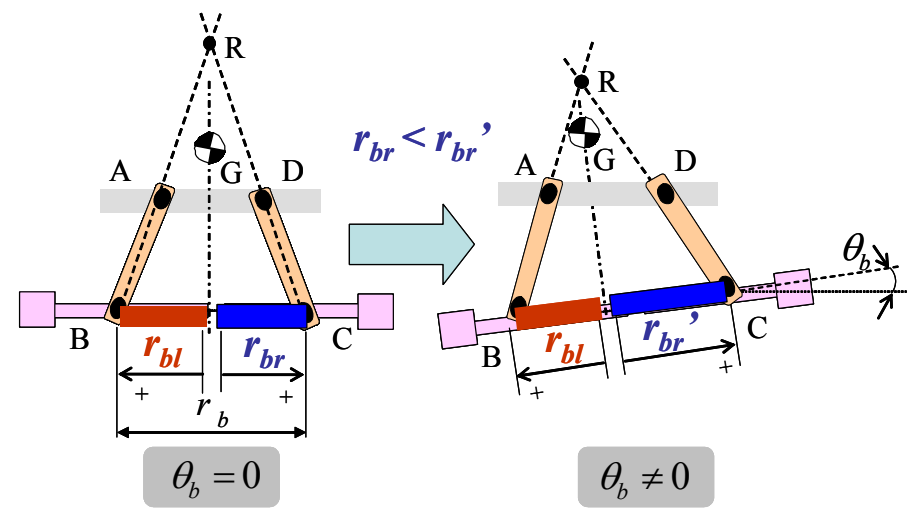

Fig. 9 Difference of link geometrical shapes

Figure 9 shows that center of gravity $\boldsymbol{G}$ and center of rotation $\boldsymbol{R}$ determine the motion 
of the center of gravity as pendulum motion. As a result, an existing link shape can realize the desired tilt angle.

In the present paper, the tilt angle is controlled by modulating the length $\mathbf{B C}$ of the car body side within the four-link mechanism, and by changing the position $\mathbf{B}[\mathbf{C}]$, it is the reduction compatible in both the car body lateral acceleration, as felt by the passengers, and the wheel load ratio. The initial state of the four-link system forms a symmetrical trapezoid, and the middle point of length $\mathbf{B C}$ is standard. The right and left lengths, $\boldsymbol{r}_{\mathrm{bl}}$ and $\boldsymbol{r}_{\mathrm{br}}$, from this standard point are made to be the two control inputs.

$$
r_{b}=r_{b l}+r_{b r}
$$

In the present study, $\boldsymbol{r}_{\mathrm{bl}}$ and $\boldsymbol{r}_{\mathrm{br}}$ are not applied as the control input. Here, $\boldsymbol{u}_{\mathrm{p}}$, which is the sum of $\boldsymbol{r}_{\mathrm{bl}}$ and $\boldsymbol{r}_{\mathrm{br}}$, and $\boldsymbol{u}_{\mathrm{m}}$, which is the difference between $\boldsymbol{r}_{\mathrm{bl}}$ and $\boldsymbol{r}_{\mathrm{br}}$, are made to be the control input.

$$
\left\{\begin{array}{l}
u_{p}=r_{b l}+r_{b r} \\
u_{m}=r_{b l}-r_{b r}
\end{array}\right.
$$

\subsection{Strategy of perfect tilting control}

In the present study, control inputs $\boldsymbol{u}_{\mathbf{p}}, \boldsymbol{u}_{\mathbf{m}}$ that collectively make both the lateral acceleration of car body felt by the passengers and the wheel load ratio zero is obtained.

$$
\left\{\begin{array}{l}
\alpha_{d}=0 \\
\Delta P / P_{m}=0
\end{array}\right.
$$

Control inputs $\boldsymbol{u}_{\mathbf{p}}, \boldsymbol{u}_{\mathbf{m}}$, which satisfy $E q$. (25) using the tilt angle $\theta_{\mathrm{b} 0}(E q$. (19)), is determined as shown in the following equations. These control inputs use the linearized equation of tilting motion to compensate for over-centrifugal acceleration.

$$
\begin{aligned}
& u_{P}=\bar{F}_{0} \quad, \quad u_{m}=\frac{\alpha_{u}}{g^{2}+\alpha_{u}{ }^{2}} \bar{E}_{0} \\
& \bar{E}_{0}=-2 g\left(\sqrt{r_{a}\left(z_{a}+\frac{2 m_{t}}{m} h_{t}\right)}+z_{a}+\frac{2 m_{t}}{m} h_{t}\right) \\
& \bar{F}_{0}=r_{d}\left(1+\sqrt{r_{a}\left(z_{a}+\frac{2 m_{t}}{m} h_{t}\right)}\right)
\end{aligned}
$$

where $\bar{E}_{0}$ and $\bar{F}_{0}$ are the values, which are both determined from the tilting vehicle mechanism. If the motion of the truck is neglected, the values of $\bar{E}_{0}$ and $\bar{F}_{0}$ may be treated as constants. From the above, $\boldsymbol{u}_{\mathbf{p}}$ is constant, and this tilting system becomes a one-control-input system by input $\boldsymbol{u}_{\mathrm{m}}$. Control input $\boldsymbol{u}_{\mathrm{m}}$ depends on the over centrifugal acceleration in $E q$. (25). If the data for the over-centrifugal acceleration $\alpha_{\mathbf{u}}$ can be obtained for a running vehicle, it will be possible to control the car body tilting using a feedforward control strategy.

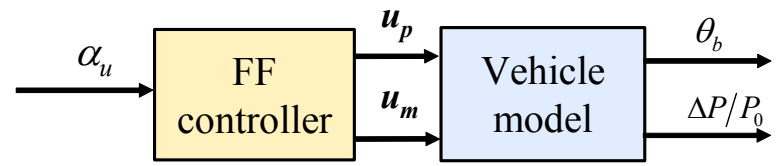

Fig. 10 Block diagram of Feedforward tilting control

\subsection{Performance of perfect tilting control}

In this section, the solution for the linear car body tilting system, which satisfies $E q$. (25), was obtained. Then, in the strict nonlinear equation of tilting motion, Eq . (6), control input $\boldsymbol{u}_{\mathrm{p}}, \boldsymbol{u}_{\mathrm{m}}$ is calculated, and the solution that satisfies $E q$. (25) is searched. 
In the nonlinear equation of motion, next search condition was regulated. The search condition set $0.01 \mathrm{~m} / \mathrm{s}^{2}$ or less in the lateral acceleration $\alpha_{\mathrm{d}}$, and $0.1 \%$ or less in the wheel load ratio.

Figure 11 shows the relationship between $\boldsymbol{r}_{\mathbf{b l}}$ and $\boldsymbol{r}_{\mathbf{b r}}$ and the relation of $\boldsymbol{u}_{\mathrm{p}}$ and $\boldsymbol{u}_{\mathbf{m}}$ by the search solution according to the nonlinear equation of motion and the perfect solution of the linear car body tilting system. The search was performed at five different running speeds, since the over-centrifugal acceleration that affected the car body by running speed, changed.

Here, $\boldsymbol{r}_{\boldsymbol{a}}=\boldsymbol{r}_{\boldsymbol{d}}=0.25[\mathrm{~m}], \rho=2.5 \times 10^{-3}[1 / \mathrm{m}], \theta_{0}=5.7[\mathrm{deg}]$

It is confirmed that the solutions are in good agreement. Thus, there exists an optimum link shape, which together makes the lateral acceleration and the wheel load ratio zero, and it is confirmed that the target of feedforward tilting control can be accurately calculated by Eq. (26).

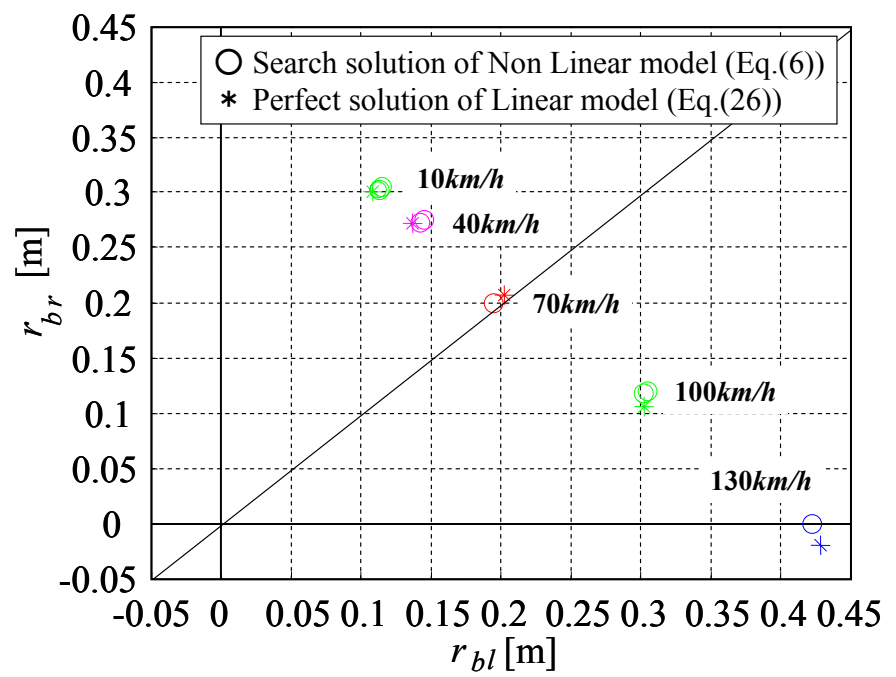

(a) Relationship of $r_{b l}$ and $r_{b r}$

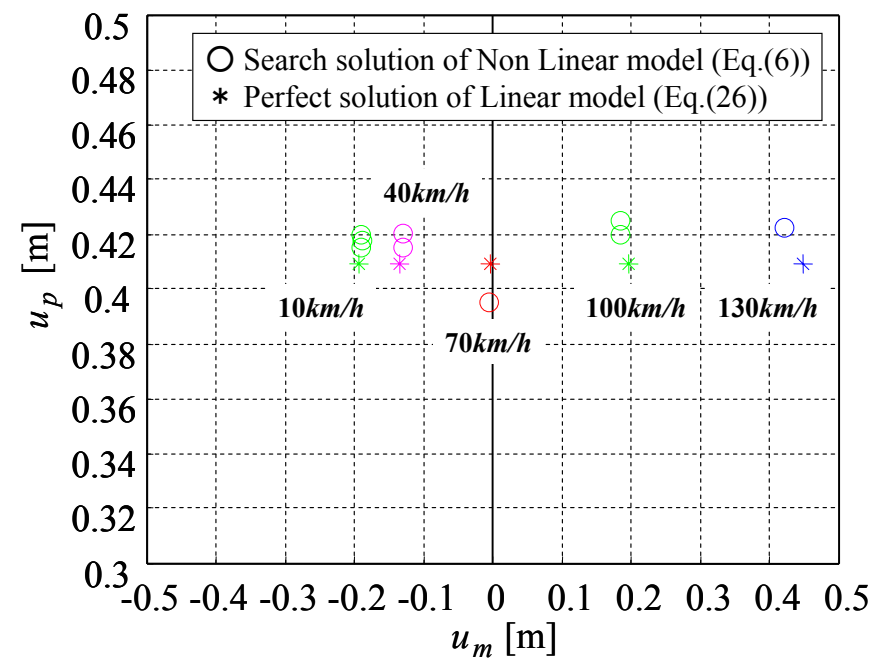

(b) Relationship of $u_{m}$ and $u_{p}$

Fig. 11 Optimal link shape of perfect tilting condition

\section{Conclusions}

In the present paper, the modeling of the tilting car body using the link mechanism was performed, and the theoretical model by the linear approximation technique was derived. Next, the effectiveness of the proposed model that was compared with the experimental results and the simulation analytical results according to theoretical model was clarified. In 
addition, a new tilting system that positively held both the lateral acceleration of the car body floor direction and the wheel load fluctuation was proposed. This tilting system does not tilt the car body compulsorily (i.e., it is not an active tilting system) and is a semi-active control system in which the balance condition of gravity and centrifugal force is varied. The following conclusions are drawn.

(1) In the theoretical model to which the linearization approximation technique was applied, it was clarified that the linear approximation is effective for realistic angles.

(2) There is a limit in order to decrease the lateral acceleration of the car body floor direction experienced by the passengers and the wheel load fluctuation by simply changing the link length and the interval length between the links of the car body tilt mechanism.

(3) By shifting joint B and C relative to the car body, the independently adjustable control system, which influenced the length between the lower-end links, was proposed.

(4) It was proven that the perfect tilting condition on the curved section that can suppress both the lateral acceleration that the passenger feels and the wheel load ratio, to be zero can be determined using the linear tilting model.

(5) By searching the solution according to the nonlinear equation of tilting motion, the effectiveness of the derived perfect car body tilting condition was clarified.

\section{References}

(1) Yukawa Y., Okamoto I., Koyanagi S., Fujimori S., Kasai K., Terada K.: An Active Tilting System for Railway Cars (In Japanese), Transactions of the Japan Society of Mechanical Engineers, Series C, Vol.53, No.496 (1987), 2588-2596

(2) Roger Goodall: Active railway suspensions : Implementation status and technological trends, Vehicle System Dynamics, 28 (1997), 87-117

(3) Matsudaira Tadashi.: Natural frequencies of passenger car and electric motor train (In Japanese), Report of railway research work, Vol.6, No.2, (1949-2), 3-14

(4) Matsudaira Tadashi.: Natural Frequencies of Lateral Vibration of Bogie Car (In Japanese), Report of railway research work, Vol.12, No.23, (1955-12), 575-585

(5) Yoshida H., Yamamoto N., Nagai M.: Tilting Control of Railway Vehicle using Electric Actuators (1st Report) (In Japanese), Transactions of the Japan Society of Mechanical Engineers, Series C, Vol.66, No.645 (2000), 1629-1635

(6) Makino H., Takano M.: Mechanical Motion (In Japanese), (1978), 54-77, Corona Publishing CO., LTD. 